\title{
Comparison of high- and low-resolution MS data for direct tissue profiling on a way from laboratory to clinic
}

\author{
A. Sorokin ${ }^{1 *}$, V. Shurhkhay ${ }^{1,3}$, K. Bocharov ${ }^{1,2}$, I. Popov $^{1,2,4}$, E. Zhvansky ${ }^{1}$, \\ A. Potapov ${ }^{3}$, E. Nikolaev ${ }^{1,2,5}$ \\ ${ }^{1}$ Moscow Institute of Physics and Technology (State University), Dolgoprudny, Russia \\ ${ }^{2}$ Institute of Energy Problems of Chemical Physics of RAS, Moscow, Russia \\ ${ }^{3}$ N.N. Burdenko Scientific Research Neurosurgery Institute, Moscow, Russia \\ ${ }^{4}$ Emanuel Institute of Biochemical Physics of Russian Academy of Sciences, Moscow, Russia \\ ${ }^{5}$ Skolkovo Institute of Science and Technology, Skolkovo, Moscow region, Russia \\ *e-mail:lptolik@gmail.com
}

Key words: high-resolution mass spectrometry, phospholipids, biomarkers, brain tumor, database

Motivation and Aim: It is known that the lipid metabolism reprogramming is one of the new hallmarks of cancer. Over the last decade, great progress was made in the understanding of the role of lipid metabolism in the progression of cancer. Highresolution mass spectrometers are widely used in research facilities but applying mass spectrometry to clinical use requires reproducing the results on the low-resolution instruments, which commonly used in routine applications. For the effective automatic classifications, the spectra of one specific type of sample have to represent the same array of peaks characterizing this type of samples. Nevertheless, in practice high- and low-resolution spectra obtained even by similar instruments from one sample are not as similar as one could expect. In this work, the feature selection approach to determine features that are common for the different resolution spectra demonstrated.

Methods and Algorithms: All experiments performed on Thermo LTQ Orbitrap XL instrument. Mass spectrometry data obtained with the novel direct-spray-from-tissue approach ion source in the negative mode as described in [1]. For registration of highresolution spectra (resolution 56,000 at $800 \mathrm{Th}$ ), we used Orbitrap analyzer. The lowresolution spectra (resolution 1,000 at $800 \mathrm{Th}$ ) we obtained using LTQ analyzer of the same instrument. All biological samples collected from dissected brain tumors during neurosurgical operations in the N.N. Burdenko Scientific Research Neurosurgery Institute. Mass-spectrometry data preprocessing and feature selection was performed in $\mathrm{R}$ environment by custom script available on request from authors.

Results: Both types of spectra from the same tumor fragment were processed separately through the data analysis pipeline for denoising, aggregation, normalization, peak picking and peak alignment. Two datasets were then aligned to each other and analyzed. The low-resolution spectra contain about one-third of the peaks, detectable in highresolution spectra; however, all major peaks found in both types of spectra. The vast majority of features distinct in tumor and unmodified brain were found in both types of spectra. More than, we have created mapping schema, which allows using classifier trained on high-resolution spectra with low-resolution spectra.

Conclusion: It is shown that low-resolution spectra preserve distinctive features of brain tumor samples and could be used in sample classification.

Acknowledgements: Supported by the RSF (16-15-10431).

\section{References}

1. Kononikhin A., Zhvansky E., Shurkhay V., Popov I., Bormotov D., Kostyukevich Y., Karchugina S., Indeykina M., Bugrova A., Starodubtseva N., Potapov A., Nikolaev E. (2015) A novel direct sprayfrom-tissue ionization method for mass spectrometric analysis of human brain tumors. Analytical and Bioanalytical Chemistry. 407(25):7797-7805. 